\title{
BioéthiqueOnline
}

\section{Art + Bioéthique : l’art comme médiation}

\section{Catherine Barnabé}

Volume 5, 2016

URI : https://id.erudit.org/iderudit/1044287ar

DOI : https://doi.org/10.7202/1044287ar

Aller au sommaire du numéro

Éditeur(s)

BioéthiqueOnline

ISSN

1923-2799 (numérique)

Découvrir la revue

Citer ce compte rendu

Barnabé, C. (2016). Compte rendu de [Art + Bioéthique : l'art comme médiation]. BioéthiqueOnline, 5. https://doi.org/10.7202/1044287ar
Résumé de l'article

Ce texte est tiré du catalogue de l'exposition Art + Bioéthique qui réunissait six artistes et six chercheurs à Espace Projet à l'hiver 2016. Il présente un survol des démarches des artistes et des oeuvres de l'exposition par le regard de l'une des commissaires. 


\title{
Art + Bioéthique : L’art comme médiation
}

\author{
COMPTE RENDU / REVIEW \\ Catherine Barnabé ${ }^{1}$
}

\section{Reçu/Received: 19 May $2016 \quad$ Publié/Published: 16 Sept 2016}

Éditrices/Editors: Lise Lévesque \& Mariana Nunez

2016 C Barnabé, Creative Commons Attribution 4.0 International License

\section{Résumé}

Ce texte est tiré du catalogue de l'exposition Art + Bioéthique qui réunissait six artistes et six chercheurs à Espace Projet à l'hiver 2016. II présente un survol des démarches des artistes et des œuvres de l'exposition par le regard de l'une des commissaires.

\section{Mots clés}

arts visuels, bioéthique, collaboration, exposition, relève artistique

\section{Summary}

This text is taken from the catalog of the exhibition Art + Bioéthique which gathered together six artists and six researchers at Espace Projet in winter 2016. It presents an overview of the artists' approaches and of the exhibited works through the eyes of one of the curators.

\section{Keywords}

visual arts, bioethics, collaboration, emerging artists, exhibition

Affiliations des auteurs / Author Affiliations

${ }^{1}$ Commissaire à Espace Projet, Montréal, Canada

Correspondance / Correspondence

Catherine Barnabé, expo.espaceprojet@gmail.com

Conflit d'intérêts

Aucun déclaré

\section{Conflicts of Interest}

None to declare

\section{Introduction}

L'exposition Art + Bioéthique s'est déroulée du 24 février au 20 mars 2016 à Espace Projet (Montréal, Canada), un organisme culturel qui présente le travail d'artistes de la relève en arts visuels et en design.

En choisissant de jumeler un artiste professionnel de la relève et un jeune chercheur en bioéthique, les organisateurs ont voulu leur permettre de sortir chacun de leurs zones de confort et de tenter le dialogue. Dialogue qui se voulait ouvert à la confrontation des regards tout comme à la convergence des idées. Des deux côtés, il s'agissait de plonger dans des situations inédites et inhabituelles. Ainsi, le projet était expérimental, et a en effet comporté son lot de surprises.

Le projet Art + Bioéthique en a été un d'échanges et de rencontres sur tous les plans. D'abord, la collaboration entre les différents acteurs derrière le projet a permis de construire un pont entre les arts et les sciences et d'imaginer une forme hybride de collaboration, créant un précédent pour les deux domaines. Puis, la forme du projet a facilité le transfert de connaissances entre les arts et les sciences de façon multidirectionnelle [1]. Enfin, les œuvres issues du projet ont agi comme des outils de médiation qui ont permis de faire des liens entre la recherche scientifique et les publics [2].

Ce texte faisant office de compte-rendu d'exposition adopte une approche externe et analytique des œuvres exposées en regard des pratiques et des démarches de chaque artiste. II repositionne les œuvres dans un contexte plus large, les détachant un peu de leur cadre de réalisation, c'est-à-dire de l'influence des travaux des chercheurs et permettant ainsi un regard artistique plus englobant. 


\section{Stephanie Coleman - Hiérarchie d'un système}

Stephanie Coleman s'intéresse au vivant et à son environnement. Elle se questionne sur les relations de l'humain à ce qui l'entoure, mais elle s'interroge aussi sur les liaisons hiérarchiques qu'il entretient avec les autres éléments du vivant. Elle tente de contrecarrer ces rapports en adoptant une perspective qui s'attarde plutôt à la place que chaque élément, chaque particule, occupe et aux relations d'interdépendance qui les lie. Esthétiquement, le fil conducteur de la pratique artistique de Coleman est le langage associé aux textiles : motifs, répétitions, tapisserie, ornements. Les médiums qu'elle utilise varient (dessin, peinture, objets trouvés, installations), mais ces préoccupations pour le détail et la matérialité de l'œuvre sont toujours présentes. Cet intérêt pour la fibre n'est pas anodin puisque l'humain entretient avec elle un rapport sensoriel de proximité (entre le vêtement et la peau), mais également un rapport mémoriel souvent lié à des souvenirs personnels ou à l'histoire plus générale des textiles. C'est donc en explorant le corps comme un territoire influencé par son environnement, où évoluent plusieurs microrelations, que Coleman représente de façon abstraite des systèmes d'interdépendance.

En collaborant avec Mathieu Noury [3], elle s'est attardée au corps vécu et sensible (à son environnement et à ce dont il est constitué) versus le corps objectivé dans un contexte médical. Elle a ainsi créé une tapisserie qui rappelle à la fois des motifs floraux et organiques : un paysage complexe et répétitif qui reprend l'idée du système composé par le vivant avec toutes ces interrelations entre les espèces et celui qui constitue le corps [4]. Les lignes qui se rejoignent et sortent du papier, les lumières qui s'allument au toucher, les éléments isolés dans les boites de Petri : tous ces morceaux interdépendants créent un système singulier et autonome, sensible et froid à la fois. La question de l'échelle est ici intéressante puisque nous pouvons nous trouver devant des microéléments ou une composition plus englobante. Le motif qu'elle répète n'est pas parfait, il comporte ses failles car il est reproduit à la main. La tapisserie a cela d'intéressant puisqu'elle permet à l'humain de proposer une représentation contrôlée du vivant. Les motifs des tapisseries sont souvent floraux ou organiques, ils sont utilisés à des fins strictement décoratives et sont l'expression, pour Coleman, d'une emprise de I'humain sur son environnement. Une préoccupation qui trouve une place centrale dans le travail de l'artiste est ce contrôle exercé par l'humain sur les autres éléments d'un système dont il fait partie, qu'il ne dirige pas, mais dont il n'est qu'une petite parcelle. En utilisant des éléments de la nature de façon contrôlée, Coleman témoigne d'un système hiérarchique qu'elle tente aussitôt de briser. En excluant l'humain de ses représentations, elle affirme la complexité du système du vivant et remet en perspective la posture anthropocentrique.

\section{Audrey Kinkead - La juste place}

Plus que ne le serait un médium ou une discipline, c'est l'animal qui est au centre de la démarche artistique d'Audrey Kinkead. Bien qu'il s'agisse d'une attention particulière qu'elle lui porte depuis toujours, l'animal n'est pas simplement un sujet comme un autre, mais lui permet de questionner à la fois nos relations avec l'Autre et le système hiérarchique qui nous lie aux vivants, système à l'équilibre fragile. Dans ses œuvres, elle observe le vivant, ce que l'on voit tout comme ce qui nous est invisible. Elle adopte une démarche qui se rapproche de celle d'une scientifique, c'est-à-dire qu'elle part d'une hypothèse qu'elle tente de vérifier. Elle fait des recherches terrain et se base sur des problématiques réelles, elle observe, prélève et documente afin de recréer un univers basé sur des faits et des recherches, mais qui est fantasmé, car tout de même subjectif dans son traitement. Sa posture d'artiste prédomine sur la démarche pragmatique qu'elle emprunte. Elle souhaite soulever les aspects semblables et les différences entre les êtres afin de remettre en question le système normatif hiérarchisé dans lequel le vivant évolue. Son projet L.O.V. (Laboratoire d'observation du vivant) qu'elle mène depuis quelques années consiste à emprunter la posture de l'ornithologue et à faire l'observation du pigeon biset dans son environnement. Elle enfile différentes combinaisons insolites qui semblent tout droit sorties d'un film de série B. Celles-ci lui permettent de créer une distance avec sa propre identité et d'adopter un état performatif le temps de ses observations. Sa combinaison attire parfois les regards et elle devient à son tour l'observée, renversant les rôles. 
Pour ce projet, elle a été inspirée de la notion de cocitoyenneté qu'aborde Dominick Rathwell-Deault dans son essai [5]. Les animaux citadins sont souvent considérés comme des nuisances alors que ce sont des êtres qui ont su s'adapter à la vie urbaine. Les choix de société nous convainquent de les traiter différemment des animaux domestiques et à les exclure de notre quotidien malgré leur omniprésence. Audrey Kinkead a décidé de faire autrement en offrant une adresse et une reconnaissance à une famille d'écureuils vivant sur son balcon [6]. Cette stratégie ludique ne consiste pas qu'à faire sourire, mais bien à questionner la cohabitation citadine entre différents êtres. La notion d'habitat chez l'animal est centrale et chacun doit pouvoir trouver place et espace pour vivre. La position biocentrique, contrairement à l'anthropocentrisme, propose de mettre simplement le vivant au centre et c'est ce qu'Audrey tente de faire par ses projets.

\section{Arkadi Lavoie Lachapelle - La performance comme résistance}

La pratique artistique d'Arkadi Lavoie Lachapelle s'incarne le plus souvent dans des performances, des manœuvres ou des installations. L'une des préoccupations récurrentes de son travail est l'idéologie productiviste individualiste à laquelle elle tente d'opposer une certaine résistance en la questionnant et la mettant en échec par diverses stratégies. Elle adopte un langage visuel qui tient de la célébration afin de bousculer le quotidien morne et les comportements routiniers qu'il indique. Elle provoque ainsi souvent des interactions entre les gens : soit en les faisant participer, parfois un peu malgré eux, ou en intervenant dans leur quotidien. Par exemple, avec 1600 œufs et Parlons d'œufs!, les visiteurs qui souhaitent entrer au Musée d'art contemporain de Montréal ou à la Galerie de I'UQAM doivent littéralement marcher sur des œufs qui contiennent des paillettes laissant ainsi une trace éphémère de leur passage. Ou bien, avec Jour de fête [7] en 2011, elle fait apparaître un bouquet de ballons colorés dans la fenêtre d'un bureau. Ce ne sont là que deux exemples de son travail qui est beaucoup plus dense, mais qui témoignent d'un aspect important de sa démarche. Dans ses performances ou ses installations, le rapport à l'Autre, soit par sa participation directe ou par sa simple présence, est toujours central. II nécessite donc une prise de conscience et soulève parfois des questions éthiques sur ce qui peut ou non être fait.

Plus récemment, une sensibilité pour la fragilité et sa prise de soin se dessine dans sa pratique. Ainsi, toujours en mettant en parallèle des contextes régulés et normatifs, et des situations ou des états sensibles et fragiles, elle joue sur les contrastes et la possibilité de leur coexistence. Dans ses projets, il est souvent question du vivant et de l'aller-retour constant entre un point de vue rationnel et sensible. Bien qu'étant une photographie trouvée, une première pour Lavoie Lachapelle, l'œuvre Jour de fête! n'est pas si éloignée de sa pratique régulière car elle met en évidence les différentes facettes d'une même situation. Une naissance fragile dans un environnement normalisé, une approche sensible versus les applications rationnelles de la médecine, le point de bascule si ténu entre la vie et la mort. Ici, tout est une question de point de vue.

\section{Mael Le Mée - Imaginer le réel}

Mael Le Mée vit et travaille à Bordeaux. II se nomme artiste transmédia : le préfixe trans faisant ici état du caractère mouvant du processus, du glissement d'un médium à un autre, du croisement des disciplines, d'un état médian et en constante évolution. Sa pratique artistique se veut éclectique, voguant de l'art contemporain au spectacle vivant, entre science-fiction et culture scientifique, touchant la recherche universitaire en sciences humaines et l'urbanisme.

En jouant avec le réel, il questionne le rapport technologique aux corps et au vivant. II souligne les notions de contrôle et d'ordre social en tentant de cerner les limites véritables des possibilités technologiques dans la création de fictions. Par exemple, avec le projet de l'Institut Benway, il propose la création sur mesure d'organes de confort. Cette entreprise de "fiction biotechnologique » engage une réflexion sur les possibilités des biotechnologies futures et sur notre rapport au corps dans une société du contrôle et de l'individualisme. Puis, avec un projet comme frUSBits \& légUSBmes, il connecte des fruits et des légumes à des ordinateurs via leur port USB, les chargeant 
symboliquement de données. Au final, on peut par exemple, boire le jus d'une orange ayant filtré un fichier MP3 tout en écoutant ledit fichier transformé. Ces propositions ont en commun de questionner notre rapport au vivant et de pousser les limites du possible. Les situations imaginées par Le Mée sont souvent ludiques car elles proposent de porter à l'extrême des avancées technologiques. C'est cette stratégie narrative qui permet de les donner à voir et de pouvoir envisager leurs potentiels afin de tenter de mieux les prendre en charge. II dessine ainsi la possibilité de se réapproprier ces technologies qui bien souvent nous dépassent.

L'œuvre qu'il propose ici rejoint ces préoccupations et permet concrètement de faire l'expérience d'une forme hybride, composite, mutante [8]. L'artiste et sa consœur outre-mer s'inventent un corps commun, une nouvelle identité qui défie les possibles. Ils expérimentent cet être ; exerçant tour à tour un certain contrôle sur l'Autre, incarnant chacun la présence physique et l'image virtuelle, s'abandonnant à cet effet de présence et à sa fugacité. Alors que l'œuvre prend son sens dans le moment des performances, elle se décline tout de même en divers éléments qui la cernent et la documentent. La création de cet être fictionnel interroge notre rapport avec le hors-norme et la différence.

\section{Karine Turcot - Illustration sémantique}

La pratique artistique de Karine Turcot questionne la façon dont nous qualifions ce qui nous entoure. Elle s'intéresse à notre rapport au monde notamment par les relations que l'on entretient avec les autres êtres vivants. Souvent, elle le fait par l'utilisation de l'animal mort dont elle se sert dans ses performances ou ses images. Elle se penche sur les définitions (individuelles et sociales) que nous attribuons et tente de les remettre en question en utilisant différentes formes de langages (plastique, narratif ou textuel). Elle ne se limite pas à un médium (sérigraphie, installation, sculpture, photographie, animation, livre, dessin, céramique, scénographie, performance) et tente toujours de soulever les différences de perceptions selon la posture adoptée et la situation.

Les recherches d'Annie Carrier et Damien Contandriopoulos portent sur les transferts de connaissance. Le partage du savoir issu du monde scientifique sert à améliorer la qualité de vie et à contribuer au mieux-être des populations. Essentielles, les stratégies de transfert ne sont pas toujours efficaces et ces failles peuvent engendrer des impacts négatifs. Pourtant, il y a des enjeux éthiques alors que l'on aborde la question de l'utilisation de stratégies potentiellement plus efficaces (marketing, psychologie cognitive) dont le format entre en contradiction avec la culture scientifique. La posture sociale du chercheur, qui est perçu soit comme un expert qui apporte des conseils ou comme un activiste qui tente de changer le monde, est à préciser. Les rôles et les impacts des différents acteurs dans ce processus de partage du savoir ainsi que les connaissances générées et l'utilisation qui en est faite posent plusieurs questions qu'Annie Carrier et Damien Contandriopoulos soulèvent.

Pour cette œuvre [9], Karine Turcot s'est inspirée d'un modèle de communications SUCCESs (Simple, Unexpected, Concrete, Credible, Emotional, Stories) dont parlent Annie Carrier et Damien Contandriopoulos dans leur essai [10] et qui est utilisé dans les milieux scientifiques, mais critiqué pour sa simplicité en regard des sujets abordés. Ce modèle est basé sur le marketing et la psychologie cognitive et tente de faciliter le transfert de connaissances scientifiques vers la population. II se décline sous des principes de base: formuler une idée simple résumant la connaissance à transmettre, la présenter de façon inattendue et concrète, avoir recours à des symboles qui établissent sa crédibilité, choisir une forme anecdotique qui permet de susciter l'émotion. Afin d'arriver à un message clair, plusieurs stratégies communicationnelles sont proposées : I'utilisation de proverbes, d'images concrètes et d'expériences, de symboles et d'histoires afin de rejoindre le public ciblé. Ici, Turcot se sert des mêmes stratégies et représente de façon caricaturale le système de santé à deux vitesses. Utilisant une mise en scène d'éléments afin de souligner les aspects du système, elle joue sur les subtilités entre les deux tableaux, qui à première vue sont semblables mais contiennent plusieurs nuances (structures des bâtiments, ornementation, éléments temporels...) qui distinguent les deux espaces. 
Avec son œuvre, Turcot souligne la complexité d'offrir un discours qui sera compris de la même façon par tous et l'adaptation nécessaire dans la prise de parole et le transfert des savoirs. Ici, c'est une approche ludique qui est adoptée: les aspects caricaturaux des éléments choisis et la fonction interactive de l'œuvre permettent de se sentir interpellé par le discours et donc de mieux le saisir. II est question de la façon de recevoir l'information qui nous est livrée et de la comprendre. Encore une fois, il s'agit de questionner la définition des choses et de souligner la perception qui varie de l'un à l'autre.

\section{Grace Stokes - Esthétisation des savoirs biomédicaux}

Grace Stokes vit et travaille à Londres. Elle perçoit l'artiste de la même façon qu'un scientifique, c'està-dire qu'il travaille avec des symboles abstraits et tente de représenter des réalités avec divers outils. Le pont entre abstraction et considérations esthétiques que l'artiste, tout autant que le scientifique, doit construire par son parcours intellectuel est essentiel à la concrétisation de son travail. Adoptant donc cette approche, elle tente de faire bouger les frontières entre art et science en s'intéressant aux possibilités que lui offre la pratique artistique. Pour elle, l'art a la possibilité de saisir les développements technologiques pour en créer des représentations à la fois esthétiques et conceptuelles. Son travail est directement ancré dans la science puisqu'elle travaille de concert avec les départements de cancérologies.

D'abord intéressée par l'esthétique des cellules, elle a su comprendre leur importance et percevoir les aspects plus fondamentaux que sont les impacts scientifique, éthique et génétique qu'elles ont, tout comme les aspects social, politique et économique qu'elles peuvent faire émerger. Dans ses travaux passés, elle a travaillé avec la Cellule HeLa : première lignée cellulaire éternelle. Celle-ci a été prélevée, sans son consentement, sur la tumeur cancéreuse de Henrietta Lack, décédée en 1951 des suites de sa maladie. II s'agit probablement de la cellule qui a le plus de valeur (monétaire et pour la recherche) et Stokes s'intéresse aux dilemmes moraux que cela engendre. Les questions liées aux maladies et aux syndromes féminins intéressent donc l'artiste depuis longtemps et ce projet en est une autre expression.

Dans ses œuvres précédentes, ces questions ont été représentées par des images agrandies de microscope, par des impressions en trois dimensions de cellules, ou encore par la reproduction en textile de microorganismes, de cellules ou de tumeurs. En étant jumelée avec Victoria Doudenkova [11], Grace Stokes a pu se pencher sur le syndrome des ovaires polykystiques et produire une œuvre polymorphe qui peut sembler à première vue simpliste et univoque, mais qui s'avère plutôt être une œuvre pleine de sens et engagée [12]. Ainsi, l'esthétique kitch qu'elle emprunte peut faire penser à des œuvres féministes du début du XXe siècle comme celles de Giorgia O'keefe qui reprenaient l'image de la fleur pour représenter le sexe féminin. Ici, Stokes reprend certains clichés qui lui servent de levier pour célébrer la femme dans toutes ses complexités plutôt que de l'enfermer dans des définitions circonscrites en faisant état de réalités autres qui ne sont pas réellement exposées.

\section{Conclusion}

Les artistes avaient été invités à se détacher de leurs pratiques et de leurs projets habituels pour travailler en collaboration avec les chercheurs en bioéthique. Par contre, on constate qu'ils ne se sont pas éloignés de leurs démarches et ont produit des œuvres qui sont, pour la plupart, très proches de leurs travaux antérieurs. Les propositions sont éclectiques et se rapprochent plus ou moins des sujets de recherche. Chacun s'est inspiré librement des échanges avec les chercheurs. Échanges qui furent en majorité fastes et nourrissants, qui pour certains mèneront même à des collaborations futures, qui pour d'autres furent l'occasion d'exposer leur pensée, de discuter et de se confronter à des visions et des raisonnements différents. L'exercice s'est avéré concluant : il a permis, d'une part, de révéler les fossés qu'il peut y avoir entre les procédés intellectuels et les perceptions des chercheurs de 
domaines éloignés, et d'autre part de mettre en lumière tous les croisements possibles tant dans les thématiques qui émergent des travaux que dans les sensibilités individuelles.

Pour Espace Projet, cette exposition représente la première réelle collaboration avec un autre domaine. Elle concrétise notre désir de créer des rencontres et de faire sortir les pratiques de leur droit chemin. II s'agit d'un projet qui s'inscrit directement dans nos aspirations futures et qui nous permet d'y voir des possibilités infinies d'échanges entre les champs de recherches et de projets inédits. Notre organisme, en se positionnant comme étant ouvert à ce type de rencontres, enclenche ainsi une nouvelle aire d'activités qui sera axée sur les collaborations entre les gens, les domaines et les structures.

\section{Références}

1. Cloutier M. Faire dialoguer les cultures: rencontre entre la bioéthique et l'art contemporain. BioéthiqueOnline. 2016;5/30.

2. Lorrain A. Art + Bioéthique : Exposition et évènements sous le signe de la collaboration entre l'éthique et l'esthétique. BioéthiqueOnline. 2016;5/31.

3. Noury M. Na-no-body: De l'oubli du corps sensible en nanomédecine. BioéthiqueOnline. 2016;5/20.

4. Coleman S. Na-no-body. BioéthiqueOnline. 2016;5/19.

5. Rathwell-Deault D. L'animal un co-citoyen, et pourquoi pas? BioéthiqueOnline. 2016;5/24.

6. Kinkead A. 54 rue DuBalcon. BioéthiqueOnline. 2016;5/23.

7. Lavoie-Lachapelle A. Jour de fête! BioéthiqueOnline. 2016;5/17.

8. Le Mée M. Mael-e(s)t-France. BioéthiqueOnline. 2016;5/27.

9. Turcot K. «Médecine à deux vitesses à la manière de SUCCESs » ou "Deux poids deux mesures ou Quod licet lovi, non licet bovi c'est-à-dire "ce qui est permis à Jupiter ne l'est pas aux vaches" „ BioéthiqueOnline. 2016;5/25.

10. Carrier A, Contandriopoulos D. Principes de communication et rôle social du chercheur en matière de transfert de connaissances: une dualité source de questionnements éthiques. BioéthiqueOnline. 2016;5/26.

11. Doudenkova V. La bioéthique, l'art et le syndrome des ovaires polykystiques: propos impressionniste visant à réhabiliter les corps tabous et les ovaires blâmés. BioéthiqueOnline. 2016;5/22.

12. Stokes G. Constant Beauty Within and PCOS as Cacti. BioéthiqueOnline. 2016;5/21. 